ARTICLE

https://doi.org/10.1038/s41467-020-17018-6

\title{
Single-atom $\mathrm{Ni}-\mathrm{N}_{4}$ provides a robust cellular NO sensor
}

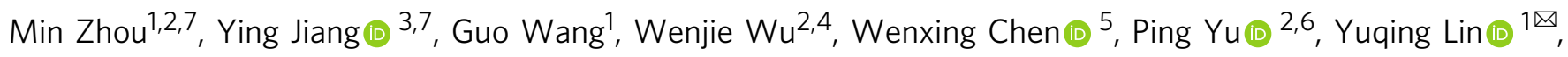
Junjie $\mathrm{Mao}^{4 \otimes}$ \& Lanqun Mao (iD ${ }^{2,6 凶}$

Nitric oxide (NO) has been implicated in a variety of physiological and pathological processes. Monitoring cellular levels of NO requires a sensor to feature adequate sensitivity, transient recording ability and biocompatibility. Herein we report a single-atom catalysts (SACs)-based electrochemical sensor for the detection of $\mathrm{NO}$ in live cellular environment. The system employs nickel single atoms anchored on $\mathrm{N}$-doped hollow carbon spheres $(\mathrm{Ni}$ $\mathrm{SACs} / \mathrm{N}-\mathrm{C}$ ) that act as an excellent catalyst for electrochemical oxidation of NO. Notably, $\mathrm{Ni}$ $\mathrm{SACS} / \mathrm{N}-\mathrm{C}$ shows superior electrocatalytic performance to the commonly used $\mathrm{Ni}$ based nanomaterials, attributing from the greatly reduced Gibbs free energy that are required for $\mathrm{Ni}$ SACs/N-C in activating NO oxidation. Moreover, Ni SACs-based flexible and stretchable sensor shows high biocompatibility and low nanomolar sensitivity, enabling the real-time monitoring of NO release from cells upon drug and stretch stimulation. Our results demonstrate a promising means of using SACs for electrochemical sensing applications.

\footnotetext{
${ }^{1}$ Department of Chemistry, Capital Normal University, Beijing 100048, China. ${ }^{2}$ Beijing National Laboratory for Molecular Sciences, Key Laboratory of Analytical Chemistry for Living Biosystems, Institute of Chemistry, the Chinese Academy of Sciences (CAS), Beijing 100190, China. ${ }^{3}$ College of Chemistry, Beijing Normal University, Beijing 100875, China. ${ }^{4}$ Key Laboratory of Functional Molecular Solids, Ministry of Education, Anhui Key Laboratory of MoleculeBased Materials, College of Chemistry and Materials Science, Anhui Normal University, Wuhu 241000, China. ${ }^{5}$ Beijing Key Laboratory of Construction Tailorable Advanced Functional Materials and Green Applications, School of Materials Science and Engineering, Beijing Institute of Technology, Beijing 100081, China. ${ }^{6}$ School of Chemical Sciences, University of Chinese Academy of Sciences, Beijing 100049, China. ${ }^{7}$ These authors contributed equally:

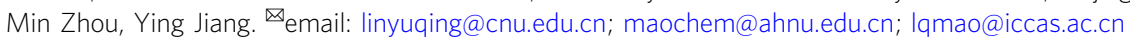


$\mathrm{N}$ itric oxide, as one of the most important signaling molecule endogenously generated by cells, functions within the cell or penetrates cell membranes to affect adjacent cells. NO plays major roles in multiple physiological and pathological processes, with the controlled release essential to maintaining vascular homeostasis ${ }^{1-5}$. An overproduction of NO in endothelial cells resulted from exposing mechanical forces, for example, circumferential stretch or fluid shear stress, triggers cascading biological reactions that dysregulates oxidative homeostasis, and causes a range of diseases, including neurodegenerative diseases, autoimmune processes and cancer6. Consequently, there has been enormous interest in the development of real-time sensing platforms for NO in normal and pathological conditions, upon mechanical stimulation of cells. Currently, flexible electrochemical sensors featuring excellent stretchability and conductivity have demonstrated to be promising tools to realize real-time monitoring of chemical signals from cells or tissues induced by deformation ${ }^{7,8}$. These sensors usually composed of nanomaterials as electrode materials and flexible polydimethylsiloxane (PDMS) substrate, yielding hybrid scaffold with synergetic functions and better electrochemical properties ${ }^{9-11}$. However, their practical applications are greatly limited by the requirement of noble metal components for efficient catalytic reactions, arguable catalytic mechanism and complicated preparation processes to maximize the utilization of the catalytic sites. Moreover, the transient nature of $\mathrm{NO}$ poses additional design challenges of sensing.

Single-atom catalysts (SACs), an emerging heterogeneous material with isolated metal atoms dispersed on solid supports, have received extensive interests since the early demonstration by Zhang et al. ${ }^{12-14}$. Owing to its optimal atom utilization, SACs offer a unique opportunity to rationally design catalysts with excellent activity, selectivity and stability compared with the stateof-the-art nanoparticles based catalysts ${ }^{15,16}$. Aided by significant progresses in synthetic, characterization, and computational modeling techniques, a substantial body of SACs-based research was centered on their application in heterogeneous catalysis field, ranging from the energy conversion to the environmental protection ${ }^{17-19}$. Surprisingly, the biological application of SACs has relatively been less exploited, probably owing to the challenges in controlling over the catalytic activities and biocompatibility of SACs in complexed biological environment. Recently, SACs have been demonstrated to exhibiting excellent performance in enzyme-like catalysis, electrochemical/chemiluminescence sensing and tumor therapy ${ }^{20-28}$, highlighting its great potency in applications in natural living system.

Herein, we present the SACs-based electrochemical sensor for the detection of NO in live cellular environment. The system features strategically designed nickel single atoms anchored on $\mathrm{N}$ doped hollow carbon spheres that act as an excellent catalyst for electrochemical oxidation of NO. Notably, Ni SACs/N-C shows superior electrocatalytic performance to the commonly used $\mathrm{Ni}$ based nanomaterials, attributing mainly from the greatly reduced Gibbs free energy that are required for Ni SACs in activating NO, a mechanism supported by the density functional theory calculation. Importantly, the $\mathrm{Ni} \mathrm{SACs} / \mathrm{N}-\mathrm{C}$ based flexible and stretchable electrochemical sensor shows high biocompatibility, reproducible mechanical compliance, and low nanomolar sensitivity, enabling real-time monitoring of trace amount of NO release from endothelial cells upon drug and stretch stimulation. As far as we know, the development of an easy, stable and even quantitative platform to analyze chemical signals in the environment of living cells has never been achieved before using SACs. This study provides important insights into the design of SACsbased electrochemical platform for cellular sensing applications, and thus broadens the practical applications of SACs.

\section{Results}

Synthesis and characterization of $\mathrm{Ni}$ SACs/N-C. The nickel single atoms anchored on $\mathrm{N}$-doped hollow carbon spheres were designed to provide a large surface area and good electrical conductivity. As depicted in Fig. 1a, the synthesis of Ni SACs/N$\mathrm{C}$ consists of several steps. Briefly, $\mathrm{SiO}_{2}$ sphere as a template was dispersed in the mixed solution of dopamine hydrochloride and $\mathrm{Ni}(\mathrm{acac})_{2}$. After stirring, polydopamine and nickel acetylacetonate were coated on the surface of the $\mathrm{SiO}_{2}$ sphere to form a core-shell $\mathrm{SiO}_{2} @$ polydopamine/ $\mathrm{Ni}(\mathrm{acac})_{2}$ structure. The structure was then pyrolyzed at a high temperature in an inert atmosphere and etched with sodium hydroxide to remove $\mathrm{SiO}_{2}$ sphere template. By careful controlling of the $\mathrm{Ni}$ weight percent and pyrolysis temperature, $\mathrm{Ni}$ SACs/N-C nanospheres can be successfully obtained.

The aberration-corrected high-angle annular dark-field scanning transmission electron microscopy (HAADF-STEM) (Fig. 1b) and transmission electron microscopy (TEM) images (Fig. 1c, d) of $\mathrm{Ni} \mathrm{SACs} / \mathrm{N}-\mathrm{C}$ revealed the hollow spherical structures of $\mathrm{Ni}$ $\mathrm{SACs} / \mathrm{N}-\mathrm{C}$, which remain the structure of the hollow $\mathrm{N}$-doped porous carbon spheres (N-C), as shown in Supplementary Fig. 1. No nanoparticles were observed among the carbon spheres, indicating that the atomic Ni sites may be embedded uniformly in the whole carbon spheres. To gain more structural information of the as-synthesized $\mathrm{Ni}$ atoms, spherical aberration correction scanning transmission electron microscope (AC-STEM) was performed. Ni atoms with a high atomic number are shown as bright dots (Fig. 1e, f) with atomic dispersed ones highlighted by red circles. Figure $1 \mathrm{~g}$ shows the energy dispersive X-ray spectroscopy (EDX) elemental mapping of the $\mathrm{Ni}$ SACs/N-C, where elements $\mathrm{C}, \mathrm{N}$, and $\mathrm{Ni}$ were homogeneously distributed over the entire architecture. No diffraction peak of $\mathrm{Ni}$ was observed in the X-ray powder diffraction (XRD) pattern for the Ni SACs/N-C (Supplementary Fig. 2), indicating that $\mathrm{Ni}$ atoms were anchored on the carbon spheres with even distribution. The binding spectrum of nitrogen was measured by XPS (Supplementary Fig. 3a), which revealed the coexistence of pyridinic $(398.4 \mathrm{eV})$, pyrrolic $(400.4 \mathrm{eV})$ and graphitic $(401.4 \mathrm{eV})$ nitrogen species. The pyrrole- $\mathrm{N}$ species is the main anchoring point for stabilizing single atomic Ni because of the strong coordination affinity. For the C1s spectrum in Supplementary Fig. 3b, three peaks with binding energies at 284.5, 286.3, and $287.9 \mathrm{eV}$ were attributed to the graphitic $\mathrm{C}, \mathrm{C}-\mathrm{O}$ and $\mathrm{C}=\mathrm{O}$, respectively, indicating the oxygen-containing property of the Ni SACs/N-C. The Ni nanoparticle immobilized on $\mathrm{N}$-doped porous carbon spheres material (Ni NPs/N-C) was fabricated as control material. As shown in Supplementary Figs. 4 and 5, Ni NPs/N-C was made up of $\mathrm{Ni}, \mathrm{N}, \mathrm{C}, \mathrm{O}$ elements and formed by random aggregation of nickel atoms during pyrolysis because exogenous nickel atoms cannot bind with nitrogen.

To further explore the electronic structure and coordination environment of $\mathrm{Ni}$ species, $\mathrm{X}$-ray absorption near-edge structure (XANES) and extended X-ray absorption fine structure (EXAFS) spectroscopy were performed. Figure 2a shows the XANES curves of $\mathrm{Ni}$ K-edge for $\mathrm{Ni} \mathrm{SACs} / \mathrm{N}-\mathrm{C}$, with $\mathrm{NiO}$ and $\mathrm{Ni}$ foil as references. The position of absorption edge of Ni SACs/N-C was located between those of $\mathrm{Ni}$ foil and $\mathrm{NiO}$, indicating that the valence state of $\mathrm{Ni}$ in $\mathrm{Ni} S A C s / \mathrm{N}-\mathrm{C}$ was between $\mathrm{Ni}^{0}$ and $\mathrm{Ni}^{\mathrm{II}}$. Fourier transform (FT) EXAFS of Ni SACs/N-C was shown in Fig. 2b. Compared with $\mathrm{Ni}$ foil and $\mathrm{NiO}, \mathrm{Ni}$ SACs/N-C exhibited only one dominant peak at $1.32 \AA$, which can be attributed to the first coordination shell of $\mathrm{Ni}-\mathrm{N}$. No obvious $\mathrm{Ni}-\mathrm{Ni}$ peaks in the FT-EXAFS spectrum of Ni SACs/N-C were observed, revealing the atomic dispersion of $\mathrm{Ni}$ in the hollow carbon support. As shown in Fig. 2c and Supplementary Table 1, the quantitative structural parameters of $\mathrm{Ni}$ in $\mathrm{Ni} \mathrm{SACs} / \mathrm{N}-\mathrm{C}$ were obtained by 
a
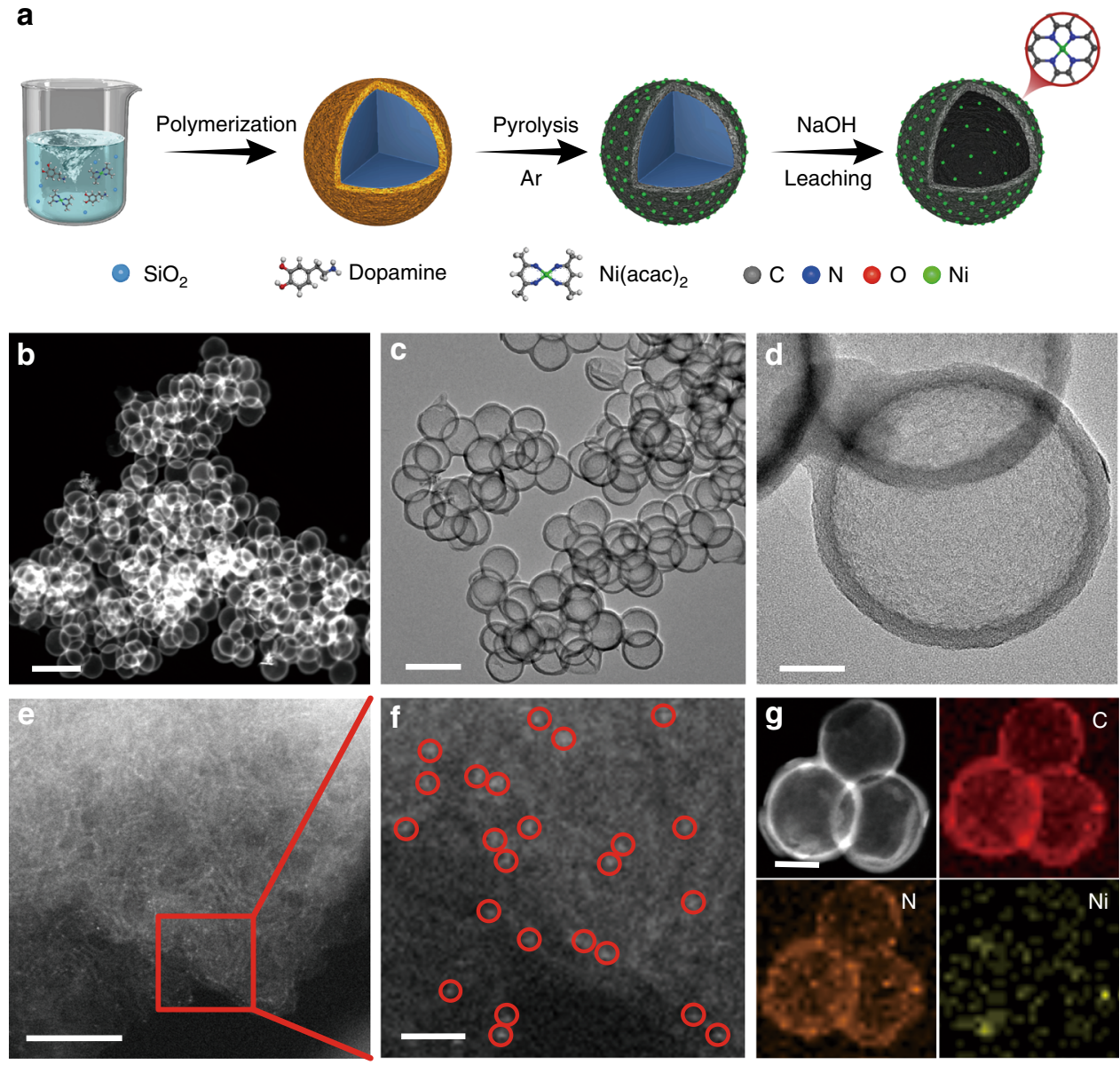

Fig. 1 Characterization of Ni SACs/N-C. a Schematic illustration of the synthesis of Ni SACs/N-C. b HAADF-STEM image of Ni SACs/N-C. Scale bar: $500 \mathrm{~nm}$. c, d TEM images of Ni SACs/N-C. Scale bar: $300 \mathrm{~nm}$ in (c), $50 \mathrm{~nm}$ in (d). e, $\mathbf{f}$ AC-STEM images of Ni SACs/N-C. Ni single atoms were indicated by red circles. Scale bar: $5 \mathrm{~nm}$ in (e), $1 \mathrm{~nm}$ in (f). g HAADF-STEM image and corresponding EDX element mapping of the Ni SACs/N-C: C (red), N (orange) and $\mathrm{Ni}$ (yellow). Scale bar: $100 \mathrm{~nm}$.

least-squares EXAFS fitting. The analysis shows that the coordination number of $\mathrm{Ni}$ was approximately 4 , and the average bond length of $\mathrm{Ni}-\mathrm{N}_{4}$ is $1.92 \AA$. Based on the above analysis, the local atomic structure around $\mathrm{Ni}$ can be constructed, proving that the isolated $\mathrm{Ni}$ atom is tetra-coordinated by $\mathrm{N}$ atom to form the $\mathrm{Ni}-\mathrm{N}_{4}$ structure that is anchored in the nitrogen-doped porous carbon matrix (Supplementary Table 2). The most stable coordination structure could also be determined by DFT calculation $^{29-31}$. To further confirm the geometrical aspects of $\mathrm{Ni}$ SACs/N-C prepared in this study, several models including graphene, a $(6,6)$ carbon nanotube, a $(10,0)$ carbon nanotube, $C_{60}$ and phenanthroline with different curvatures were proposed as carbon substrate for the $\mathrm{Ni}-\mathrm{N}_{4}$ structure. Theoretically calculated spectra are almost consistent with the experimental ones. It is likely that the models proposed with the carbon structures mentioned above as the substrate for the $\mathrm{Ni}-\mathrm{N}_{4}$ structure may all exist in the experiments, of which the most possible one is the graphene model, as shown in Fig. 2d and Supplementary Fig. 6.

Electrochemical performance and mechanistic study of $\mathrm{Ni}$ SACs/N-C-catalyzed NO oxidation. The electrochemical oxidation of NO is a promising technology for exploring the multiple roles of NO in biological system, in which hunting for effective catalysts for NO oxidation is the main challenge for highly sensitive and efficient determination of NO. Having prepared and characterized the atomically dispersed Ni SACs/N-C, we then set out to study its electrocatalytic ability towards NO oxidation. To this end, we modified the catalyst on a glassy carbon electrode for all the subsequent testing. Electrochemical oxidation of $\mathrm{NO}$ on $\mathrm{Ni}$ SACs/N-C was measured by cyclic voltammetry (CV), as shown in Fig. 3a. Ni SACs/N-C exhibits excellent activity towards $\mathrm{NO}$ oxidation that commences at $\mathrm{ca}$. $+0.60 \mathrm{~V}$ and researches a welldefined oxidation peak at $+0.83 \mathrm{~V}$ (red curve). These potentials are more negative than those at Ni NPs/N-C (blue curve) and $\mathrm{N}$ $\mathrm{C}$ (green curve), demonstrating the higher catalytic activity of $\mathrm{Ni}$ SACs/N-C. Moreover, the peak current for $\mathrm{NO}$ oxidation at $\mathrm{Ni}$ $\mathrm{SACs} / \mathrm{N}-\mathrm{C}$ was much higher than those at Ni NPs/N-C and N-C, as shown in Fig. 3b. It's worth noting that in these experiments, the amounts of $\mathrm{Ni}$ (by weight) modified onto the electrode in $\mathrm{Ni}$ $\mathrm{SACs} / \mathrm{N}-\mathrm{C}$ and $\mathrm{Ni} \mathrm{NPs} / \mathrm{N}-\mathrm{C}$ were controlled to be the same. Therefore, the observed better catalytic performance of $\mathrm{NO}$ oxidation from Ni SACs/N-C was most probably resulted from the atomically dispersed catalytic sites. Ni SACs/N-C has higher turnover frequency (TOF) value of $1.23 \times 10^{4} \mathrm{~h}^{-1}$ than Ni NPs/ $\mathrm{N}-\mathrm{C}$ at the applied potential of $+0.85 \mathrm{~V}$ (Supplementary Fig. 7a), again suggesting the better catalytic performance toward $\mathrm{NO}$ oxidation of Ni SACs/N-C. The Ni SACs/N-C based sensor also has a good durability (Supplementary Fig. 7b), validating its application for continuous sensing of NO. In addition, we investigated the $\mathrm{NO}$ adsorption behavior on the SACs/N-C using Fourier-transform infrared (FTIR) spectroscopy and found that the adsorption of $\mathrm{NO}$ on $\mathrm{Ni}$ site belongs to the top but not to the bridged type (Supplementary Fig. 7c), implying that the 

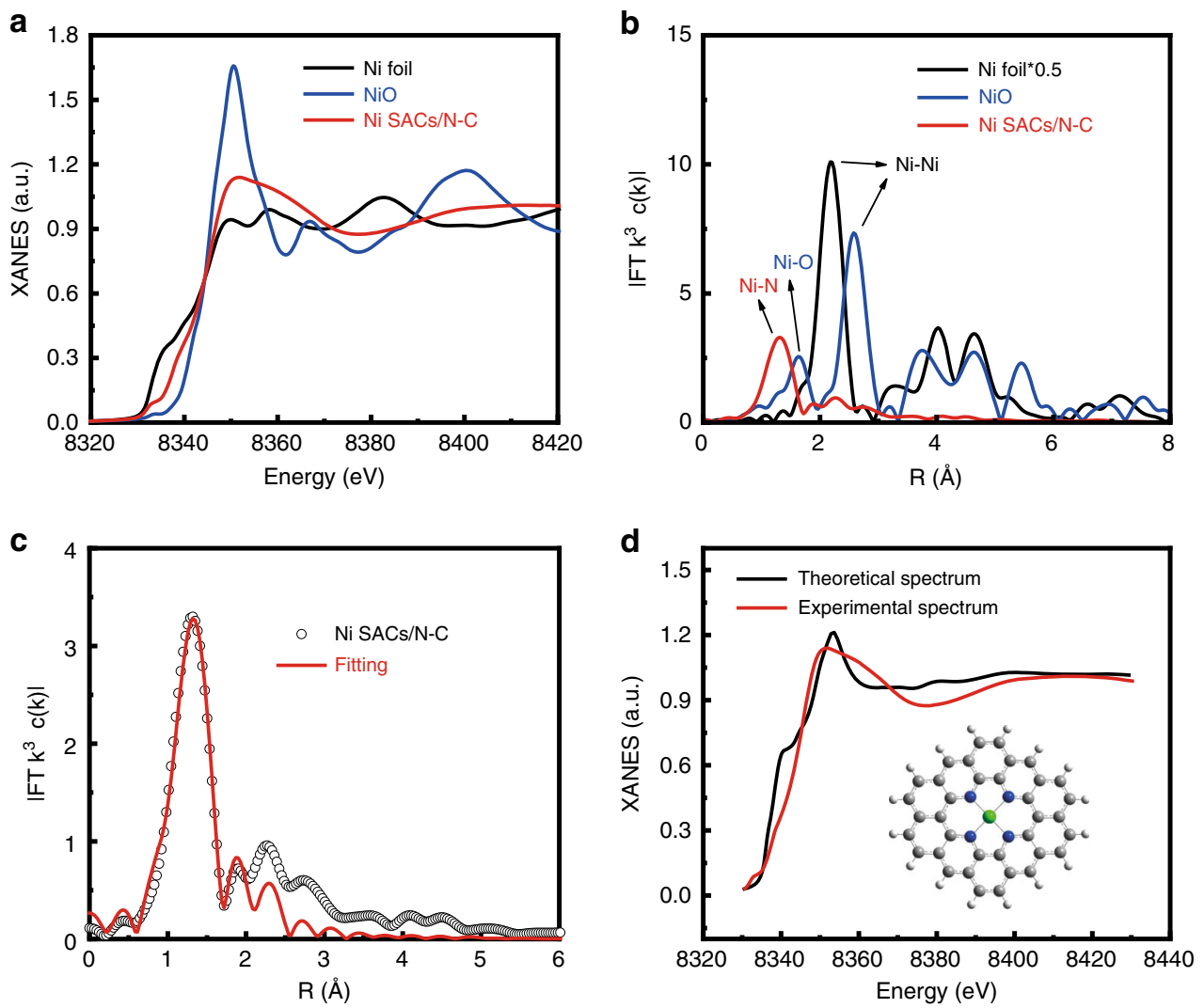

Fig. 2 Structural analysis of Ni SACs/N-C. a XANES spectra of Ni SACs/N-C. b Fourier transforms of the EXAFS spectra for the Ni K-edge of Ni SACs/N$\mathrm{C}, \mathrm{NiO}$ and $\mathrm{Ni}$ foil. $\mathbf{c}$ The corresponding EXAFS fitting curves of the Ni SACs/N-C nanospheres at R space. $\mathbf{d}$ Comparison between the XANES experimental spectrum for graphene models of Ni SACs/N-C at Ni K edge (red curve) and the theoretical spectra calculated with the depicted structures (black curve). Inset, proposed $\mathrm{Ni}-\mathrm{N}_{4}$ architectures.

single-atom state of $\mathrm{Ni}$ was maintained after $\mathrm{NO}$ adsorption. We also found that the modification of Nafion on the sensor surface well suppressed the current response toward nitrite $\left(\mathrm{NO}_{2}{ }^{-}\right)$ (Supplementary Fig. 8), which acts as the main interference toward NO detection ${ }^{32}$.

Having demonstrated the excellent catalytic activity of Ni SACs for the electrooxidation of NO, we moved on further to perform mechanistic study by using DFT calculation. It is generally considered that in a typical catalytic reaction, $\mathrm{NO}$ molecule first loses one electron to form $\mathrm{NO}^{+}$, as reported previously ${ }^{9}$. However, this is an energetically unfavorable step since the first ionization energy of NO molecule is higher than $9 \mathrm{eV}$. Here, we proposed the following mechanism (Eqs. (1) and (2) to describe the electrocatalytic reaction, in which the Eq. (1) refers to reaction from step 1, whereas Eq. (2) refers a total reaction from consecutive step 2 to 5 as shown in Fig. 3c. The detailed mechanism including all the proposed reactions were included and discussed in Supplementary Fig. 9,

$$
\begin{gathered}
{ }^{*}+\mathrm{NO}={ }^{*} \mathrm{NO} \\
{ }^{*} \mathrm{NO}+\mathrm{H}_{2} \mathrm{O}={ }^{*}+\mathrm{HNO}_{2}+\mathrm{H}^{+}+\mathrm{e}^{-}
\end{gathered}
$$

The * stands for the active sites in SACs, while *NO represents the catalyst with NO molecule adsorbed. The changes in the Gibbs free energy (including the zero-point energy) in Eqs. (1) and (2) on Ni SACs/N-C (0.67 and $-0.13 \mathrm{eV}$, Fig. 3d, black curve) and on Ni NPs/N-C (-1.18 and $1.72 \mathrm{eV}$, Fig. $3 \mathrm{~d}$, red curve) were calculated, which were shown in Fig. $3 \mathrm{~d}$ and also demonstrated in Supplementary Fig. 9. We also considered the solvation effect with the Polarized Continuum Model (PCM). We found that the change of Gibbs free energy of Eq. (2) only increases by $0.08 \mathrm{eV}$, and that of Eq. (1) remains almost unchanged, both showing the minimal effect of solvation. The $\mathrm{Ni}$ atom has a $3 \mathrm{~d}^{8} 4 \mathrm{~s}^{2}$ electronic configuration, whereas its $3 \mathrm{~d}$ and $4 \mathrm{p}$ orbitals are fully occupied by the four ligands in the Ni SACs/ $\mathrm{N}-\mathrm{C}$. Thus, further coordination with a $\mathrm{NO}$ molecule is less favorable than that for Ni NPs/N-C. Since the change of Gibbs free energy for the total reaction $\mathrm{NO}+\mathrm{H}_{2} \mathrm{O}=\mathrm{HNO}_{2}+\mathrm{H}^{+}+\mathrm{e}^{-}$ is fixed, Eq. (2) is favorable for the $\mathrm{Ni} S A C s / \mathrm{N}-\mathrm{C}$, therefore reasonably explaining the excellent electrocatalytic property of $\mathrm{Ni}$ $\mathrm{SACs} / \mathrm{N}-\mathrm{C}$ towards NO oxidation as described above.

Real-time sensing of NO release with the Ni SACs/N-C-based stretchable sensor. To realize real-time sensing of $\mathrm{NO}$ release from cells under mechanical deformation, $\mathrm{Ni} S A C s / \mathrm{N}-\mathrm{C}$ was confined on a flexible PDMS substrate to form a stretchable electrochemical sensor (Fig. 4a). Ni SACs/N-C used here features both high catalytic activity and excellent electrical conductivity for NO oxidation, simplifying the sensor preparation. This is remarkable because most of the stretchable sensors reported so far have normally been prepared with composite materials respectively in charge of catalytic recognition of target and electric conducting ${ }^{10,11}$. As shown in Fig. $4 \mathrm{~b}$, the as-prepared $\mathrm{Ni}$ SACs/N-C-based stretchable electrochemical sensor is well responsive to NO with a nanomolar detection limit. The sensi-

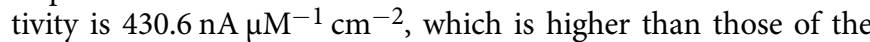
stretchable electrochemical sensors prepared with single metal nanomaterials $s^{7,33}$, carbon materials ${ }^{34}$, and even composite electrode materials $^{11,35-37}$ (Supplementary Table 3 ). We also studied the selectivity of the $\mathrm{Ni}$ SACs/N-C based sensor against 

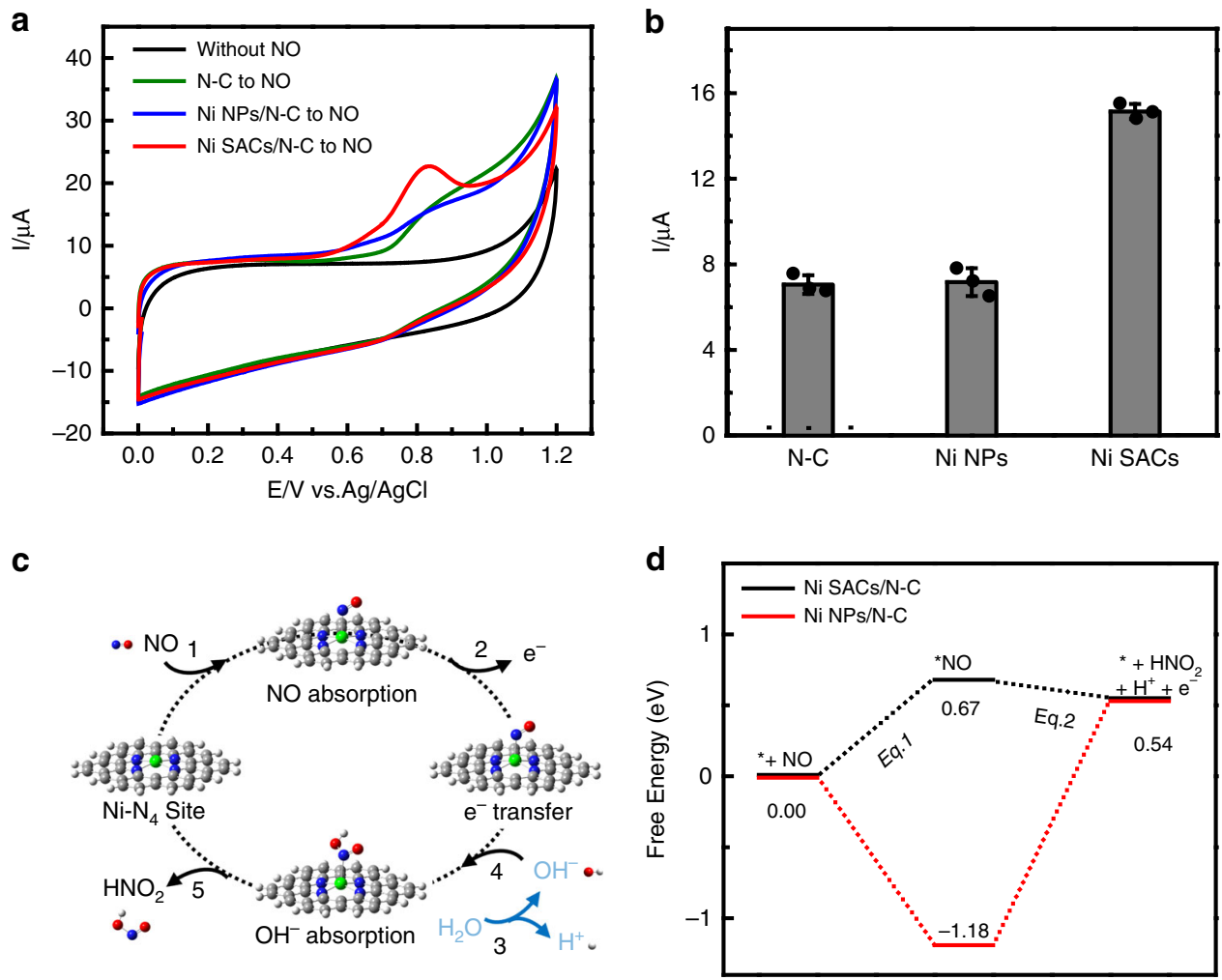

Fig. 3 Performance and mechanism of Ni SACs/N-C for electrocatalytic NO oxidation. a Cyclic voltammograms obtained at glassy carbon electrodes modified with Ni SACs/N-C (red curve), Ni NPs/N-C (blue curve) or N-C (green curve) in deaerated phosphate-buffered solution (PBS) containing $0.18 \mathrm{mM}$ NO. Black curve represents a control experiment of Ni SACs/N-C in deaerated PBS without NO. b Data are represented as current response recorded with three different modified electrodes shown in (a). Error bars=standard deviation $(n=3)$. c The proposed structures for electrocatalytic oxidation process of $\mathrm{NO}$ on Ni SACs/N-C. $\mathbf{d}$ The Gibbs free energy profile along the pathway from $\mathrm{NO}$ to $\mathrm{HNO}_{2}$.

commonly interfering species in a biological system, and found that that the sensor has excellent selectivity toward NO sensing (Supplementary Fig. 10). Figure $4 \mathrm{c}$ demonstrates the calibration curves of the sensor toward the successive addition of NO before and after 50\% stretching the sensor. The similar calibration curves were obtained, demonstrating the high tolerance to mechanical stretching of our sensor. Further tests showed that our sensor has a long-term resistance stability against mechanical bending (Supplementary Fig. 11). When the sensor was bent to a different curvature (Supplementary Fig. 11a) or for 1000 cycles with a radius of $5 \mathrm{~mm}$ (Supplementary Fig. 11b), the change of deformed resistance/original resistance $\left(R / R_{0}\right)$ remained almost negligible, showing the mechanical stability of the Ni SACs/N-Cbased sensor.

Next, we explored whether the Ni SACs/N-C-based stretchable sensor could be used to detect NO in the cellular environment, with human umbilical vein endothelial cells (HUVECs) as a model cell line. HUVECs were cultured on the surface of the stretchable sensor for $24 \mathrm{~h}$, reaching a density of $2 \times 10^{6} \mathrm{cells} / \mathrm{cm}^{2}$. Most of the cells maintained their regular shapes (Fig. 4d), showing the excellent biocompatibility of Ni SACs/N-C under the conditions employed here. Further toxicity tests showed that more than $80 \%$ of HUVECs maintain cell viability after cocultured with different concentrations of Ni SACs/N-C for $48 \mathrm{~h}$ (Supplementary Fig. 12). We then studied the capability of the Ni SACs/N-C-based sensor to monitor NO release from HUVECs under stimulation of L-arginine (L-Arg). It is known that L-Arg treatment activates the nitric oxide synthase (NOS) and induces the production of $\mathrm{NO}$ in endothelial cells ${ }^{38-40}$. As shown in Fig. $4 \mathrm{e}$ (black curve), the treatment of $10 \mathrm{mM}$ of L-Arg led to an obvious increase of the oxidation current. Moreover, when we simultaneously treated HUVECs with $10 \mathrm{mM}$ L-NGNitroarginine Methyl Ester (L-NAME), a precursor of nitric oxide synthase (NOS) inhibitor, and $10 \mathrm{mM} \mathrm{L-Arg,} \mathrm{no} \mathrm{current}$ increase was observed (Fig. $4 \mathrm{e}$, red curve), confirming the current response of the Ni SACs/N-C is due to the release of NO. In addition, the introduction of L-Arg solution $(10 \mathrm{mM})$ to the $\mathrm{Ni}$ SACs/N-C-based sensor without cells generated no perceptible current response (Fig. 4e, blue curve). Taken together, these results substantially demonstrate that the $\mathrm{Ni}$ SACs/N-C-based stretchable sensor could selectively and sensitively detect the dynamics of NO in the cellular environment.

To further demonstrate the application of the stretchable electrochemical sensor prepared with Ni SACs/N-C, the sensor incubated with HUVECs on the surface was mounted on a special bracket capable of applying precisely controlled mechanical force. When the sensor was stretched to approximately a 50\% change in the electrode area, an increase in the current signal (i.e., $520 \mathrm{nA}$ ) was observed (Fig. 4g, black curve) and the corresponding schematic cellular mechanotransduction and $\mathrm{NO}$ oxidation mechanism was proposed in Fig. 4f. In addition, much smaller current change (ca. $10 \mathrm{nA}$ ) was recorded on the Ni SACs/N-Cbased stretchable sensor without cell at $50 \%$ deformation (Fig. $4 \mathrm{~g}$, blue curve), which may be ascribed to the capacitive current change, highlighting that the mechanical force itself contributes minimally to the current response recorded with cells on the sensor surface. Moreover, when cells were pretreated with LNAME (which inhibits the generation of NO), followed by $50 \%$ mechanical force stimulation, less than $10 \%$ of the current response was observed (Fig. $4 \mathrm{~g}$, red curve), as compared with the groups without L-NAME treatment (Fig. $4 \mathrm{~g}$, black curve). It is of note that an extensive mechanical strain not only provokes 
a

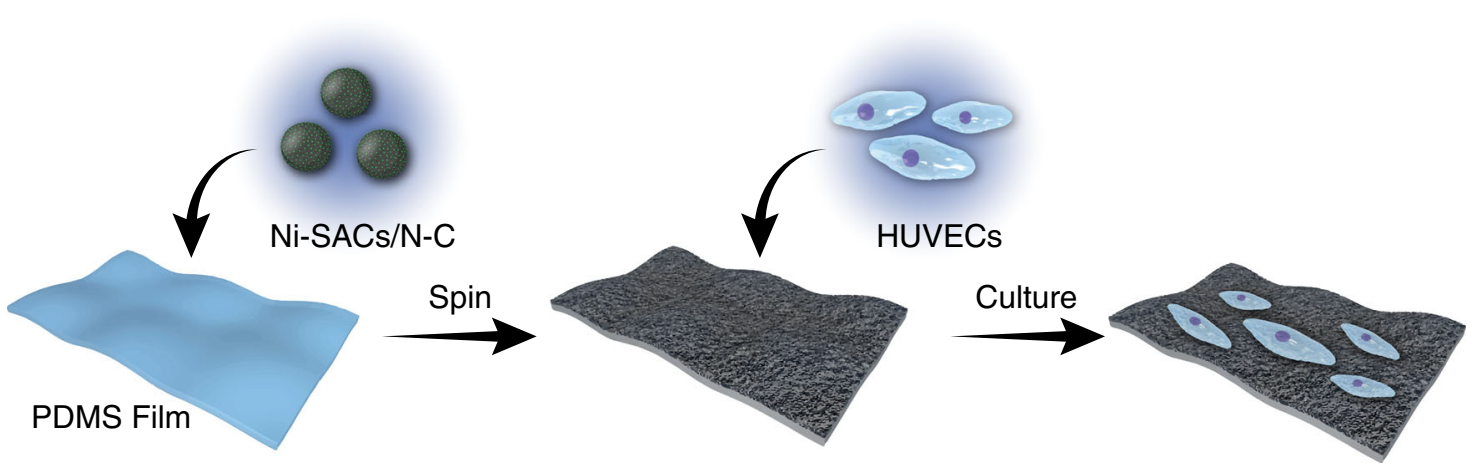

b
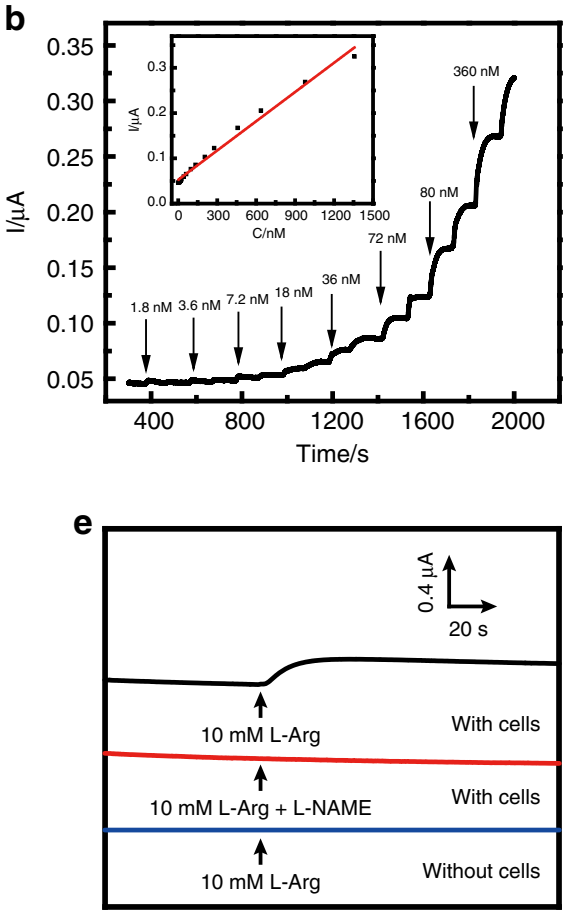

C

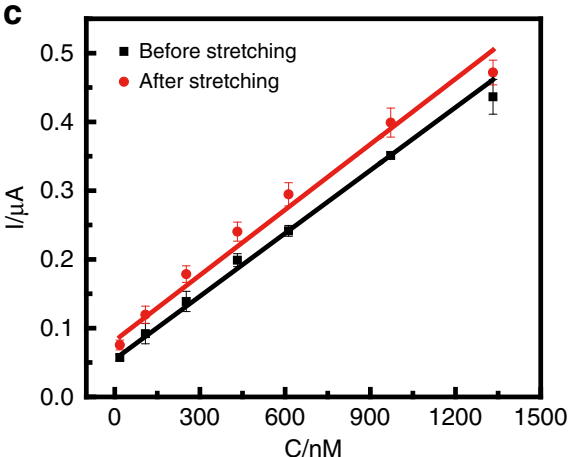

d
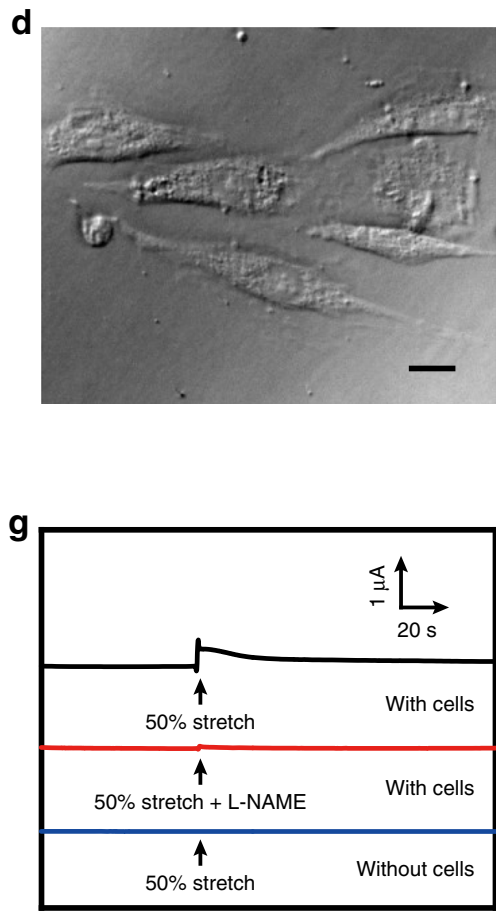

Fig. 4 Real-time detection of NO released from living cells. a Schematic illustration of the fabrication of Ni SACs/N-C-based stretchable sensor for $\mathrm{NO}$ sensing and HUVECs culturing. b Amperometric response of the Ni SACs/N-C-based stretchable sensor $\left(0.5 \mathrm{~cm}^{2}\right)$ to successive addition of NO at $+0.80 \mathrm{~V}$. Inset, calibration curve. c Calibration curves of the Ni SACs/N-C-based sensor for NO before (black curve) and after (red curve) sensor stretching. Error bars = standard deviation $(n=3)$. d Microscopic image of HUVECs cultured on the surface of the Ni SACs/N-C-based sensor for $24 \mathrm{~h}$. Scale bar: $20 \mu \mathrm{m}$. e Monitoring NO release from HUVECs subject to $10 \mathrm{mM} \mathrm{L-Arg}$ with the Ni SACs/N-C-based sensor (black curve). Red and blue curves represent the current responses under stimulation of $10 \mathrm{mM} \mathrm{L-Arg}$ and L-NAME (red curve) to cells and $10 \mathrm{mM}$ L-Arg stimulation without cells (blue curve). $\mathbf{f}$ Diagram of sensor stretching, and $\mathbf{g}$ real-time monitoring of NO released from HUVECs subject to $50 \%$ stretch moduli (black curve) with the Ni SACs/N-C-based sensor. Red and blue curves represent the current responses from the sensor under $50 \%$ stretch and L-NAME stimulation with cells (red curve) and under $50 \%$ stretch stimulation without cells (blue curve).

HUVECs to release of NO, but also triggers other signaling pathways ${ }^{41-43}$. Taken together, these results suggest that $\mathrm{Ni}$ SACs/N-C with atomically dispersed $\mathrm{Ni}-\mathrm{N}_{4}$ catalytic site presents a robust electrocatalyst for NO oxidation and sensing, making it reliable as a stretchable electrochemical sensing material in biological detection.

\section{Discussion}

In summary, by using nickel single-atom based electrocatalyst, we demonstrate that the SACs facilities NO oxidation and accomplish the real-time NO sensing in live cellular environment. DFT calculation reveals a two-step catalytic reaction mechanism involved in activating $\mathrm{NO}$ with $\mathrm{Ni} \mathrm{SACs} / \mathrm{N}-\mathrm{C}$, in which a greatly reduced Gibbs free energy process enables $\mathrm{Ni} S A C s / \mathrm{N}-\mathrm{C}$ with high electrocatalytic performance. Moreover, when the catalytic activity of the Ni SACs/N-C was integrated with a flexible sensor platform, a highly robust, biocompatible and low-nanomolar sensitive electrochemical tool was created, allowing the recording of trace amount of NO release under a physiological environment. The designing of this single-atom nickel catalyst not only provides great opportunities for live cell analysis but also sheds light on our understanding of electrocatalytic oxidation of $\mathrm{NO}$ through theoretical insights, opening a paradigm for the development of SACs-based advanced sensing device for potential healthcare monitoring.

\section{Methods}

Preparation of N-C. The preparation of different sized silica spheres involves the ammonia-catalyzed hydrolysis and condensation of tetraethyl orthosilicate (TEOS) in an aqueous ethanol solution via the classical stöber method ${ }^{44}$. To do this, 100 $\mathrm{mL}$ of absolute ethanol, $30 \mathrm{~mL}$ of deionized water, and $5 \mathrm{~mL}$ of $28 \% \mathrm{NH}_{3} \mathrm{H}_{2} \mathrm{O}$ were mixed and stirred. A total of $5 \mathrm{~mL}$ of TEOS was added into the mixture and stirred for $1 \mathrm{~h}$. Then $10 \mathrm{~mL}$ mixture of ethanol and water containing of $0.5 \mathrm{~g}$ dopamine 
monomers was added into the preceding mixture and stirred. After reaction for 12 $\mathrm{h}$, the silica spheres were isolated by centrifugation, dried at $70^{\circ} \mathrm{C}$ under vacuum, heated to $900^{\circ} \mathrm{C}$ for $3 \mathrm{~h}$ at the heating rate of $3^{\circ} \mathrm{C} / \mathrm{min}$ under flowing of Ar gas, and finally naturally cooled to room temperature. The obtained samples were etched in the aqueous solution of $4 \mathrm{M}$ sodium hydroxide for $24 \mathrm{~h}$ and collected by centrifugation, washed subsequently with water and ethanol and finally dried at $70^{\circ} \mathrm{C}$ under vacuum for overnight.

Preparation of Ni SACs/N-C. Ni SACs/N-C was synthesized with the procedures similar to those for the synthesis of N-C. Briefly, $100 \mathrm{~mL}$ of absolute ethanol, $30 \mathrm{~mL}$ of deionized water, and $5 \mathrm{~mL}$ of $28 \% \mathrm{NH}_{3} \cdot \mathrm{H}_{2} \mathrm{O}$ were mixed and stirred. A total of $5 \mathrm{~mL}$ of TEOS was added into the mixture and stirred for $1 \mathrm{~h}$. Then, $10 \mathrm{~mL}$ mixture of ethanol and water containing of $0.5 \mathrm{~g}$ dopamine monomers and $5.62 \mathrm{mg} \mathrm{Ni}(\mathrm{acac})_{2}$ was added into the preceding mixture and stirred. After a reaction time of about $12 \mathrm{~h}$, the silica spheres were isolated by centrifugation, dried at $70^{\circ} \mathrm{C}$ under vacuum, heated to $900{ }^{\circ} \mathrm{C}$ for $3 \mathrm{~h}$ at the heating rate of $3^{\circ} \mathrm{C} / \mathrm{min}$ under flowing $\mathrm{Ar}$ gas, and finally naturally cooled to room temperature. The obtained samples were etched in the aqueous solution of $4 \mathrm{~mol} / \mathrm{L}$ sodium hydroxide for $24 \mathrm{~h}$ and collected by centrifugation, washed subsequently with water and ethanol and finally dried at $70^{\circ} \mathrm{C}$ under vacuum for overnight. The $\mathrm{Ni}$ content in $\mathrm{Ni} \mathrm{SACs} / \mathrm{N}-\mathrm{C}$ determined by inductively coupled plasma optical emission spectrometry (ICP-OES) is $\sim 0.10 \%$ (wt\%).

Preparation of Ni NPs/N-C. A $56.2 \mathrm{mg} \mathrm{Ni(acac})_{2}$ and $0.1 \mathrm{~g}$ dopamine derived hollow carbon sphere were mixed and grinded. The mixture was then heated to $900^{\circ} \mathrm{C}$ for $1 \mathrm{~h}$ at the heating rate of $3^{\circ} \mathrm{C} / \mathrm{min}$ under the flowing of $\mathrm{Ar}$ gas and naturally cooled to room temperature. The obtained samples were washed with water and ethanol for 2-3 times, collected by centrifugation and finally dried at $70{ }^{\circ} \mathrm{C}$ under vacuum for overnight. The Ni content in Ni NPs/N-C determined by ICP-OES is approximately $0.85 \%$ (wt\%)

Preparation of Ni SACs/N-C based sensor. Ni SACs/N-C suspension $\left(2 \mathrm{mg} \mathrm{mL}^{-1}\right)$ was prepared by dispersing Ni SACs/N-C into mixture of deionized water and $\mathrm{N}$, $\mathrm{N}$-dimethylformamide solution under ultrasonication to form homogeneous dispersion. PDMS film ( $500 \mu \mathrm{m}$ in thickness) was obtained by spin-coating the degassed liquid prepolymer and cross-linker $(\mathrm{w} / \mathrm{w}=10: 1)$ on a plastic substrate at a spin rate of $500 \mathrm{rpm}$ for $10 \mathrm{~s}$ and thermally cured at $60^{\circ} \mathrm{C}$. Before PDMS was fully cured, Ni SACs/N-C suspension was dropped and dried at $60^{\circ} \mathrm{C}$ for $1 \mathrm{~h}$. Then, the Ni SACs/N-C/PDMS film was peeled off from the substrate and contacted with a copper wire via carbon paste. Finally, the Ni SACs/N-C/PDMS film electrode was further coated with Nafion film by dropping $4 \mu \mathrm{L} 0.5 \%$ (w/v) Nafion solution in ethanol on the electrode surface $\left(0.5 \mathrm{~cm}^{2}\right)$ and the electrode was dried in air to form Ni SACs/N-C-based sensor.

Characterization. TEM images were taken on a Hitachi HT7700 transmission electron microscope. The high-resolution TEM, HAADF-STEM images, and the corresponding energy-dispersive X-ray (EDX) mapping were recorded by a JEOL JEM-2100F high-resolution transmission electron microscope operating at $200 \mathrm{kV}$. Inductively coupled plasma optical emission spectroscopy (ICP-OES) was measured by Thermo Fisher IRIS Intrepid II. Powder X-ray diffraction patterns were measured with a Bruker D8 with $\mathrm{Cu} \mathrm{Ka}$ radiation $(\lambda=1.5406 \AA)$. The X-ray photoelectron spectroscopy (XPS) was measured by a PHI Quantera SXM system under $3.1 \times 10^{-8} \mathrm{~Pa}$ using $\mathrm{Al}^{+}$radiation at room temperature.

XAFS measurements and analysis. The X-ray absorption find structure spectra $\mathrm{Ni}$ K-edge were collected at BL1W1B station in Beijing Synchrotron Radiation Facility (BSRF). The data were collected in fluorescence excitation mode using a Lytle detector. All samples were pelletized as disks of $13 \mathrm{~mm}$ diameter using graphite powder as a binder. The Ni L-edge absorption near-edge structure spectra were collected at BL4B9B station in BSRF. The acquired EXAFS data were processed according to the standard procedures using the ATHENA module implemented in the IFEFFIT software packages. The EXAFS spectra were gained by subtracting the post-edge background from the overall absorption and then nor malizing with respect to the edge-jump step. Subsequently, the $\chi(\mathrm{k})$ data were Fourier transformed to real $(\mathrm{R})$ space using a hanning windows $\left(\mathrm{dk}=1.0 \AA^{-1}\right)$ to separate the EXAFS contributions from different coordination shells. To obtain the quantitative structural parameters around central atoms, least-squares curve parameter fitting was carried out using the ARTEMIS module of IFEFFIT software packages. The following EXAFS equation was applied (Eq. (3)):

$$
\chi(k)=\sum_{j} \frac{N_{j} S_{o}^{2} F_{j}(k)}{k R_{j}^{2}} \cdot \exp \left[-2 k^{2} \sigma_{j}^{2}\right] \cdot \exp \left[\frac{-2 R_{j}}{\lambda(k)}\right] \cdot \sin \left[2 \mathrm{k} R_{j}+\phi_{j}(k)\right]
$$

$S_{0}^{2}$ is the amplitude reduction factor, $F j(k)$ is the effective curved-wave backscattering amplitude, $\mathrm{N}_{\mathrm{j}}$ is the number of neighbors in the $j^{\text {th }}$ atomic shell, $R_{j}$ is the distance between the X-ray absorbing central atom and the atoms in the $j^{\text {th }}$ atomic shell (backscatter), $\lambda$ is the mean free path in $\AA, \phi_{j}(k)$ is the phase shift (including the phase shift for each shell and the total central atom phase shift), $\sigma j$ is Debye-Waller parameter of the jth atomic shell (variation of distances around the average $R_{j}$ ). The functions $F_{j}(k), \lambda$ and $\phi_{j}(k)$ were calculated with the ab initio code FEFF8.2.

Electrocatalytic oxidation of NO on Ni SACs/N-C. Briefly, NO was prepared by slowly dropping $4 \mathrm{M} \mathrm{H}_{2} \mathrm{SO}_{4}$ into deoxygenated $2 \mathrm{M} \mathrm{NaNO}_{2}$ solution ${ }^{7}$. A $1.8 \mathrm{mM}$ NO-saturated solution was obtained by perfusing pure NO gas into PBS for $30 \mathrm{~min}$ at $20^{\circ} \mathrm{C}$. Electrochemical measurements were performed on a computer-controlled electrochemical analyzer (CHI 660, Shanghai, China) with a three-electrode system with the $\mathrm{Ni} \mathrm{SACs} / \mathrm{N}-\mathrm{C} / \mathrm{PDMS}$ as working electrode, $\mathrm{Ag} / \mathrm{AgCl}$ as reference electrode and Pt wire as counter electrode. A 0.10 M PBS was used as the electrolyte. All electrochemical experiments were carried out under the protection of nitrogen gas atmosphere. Calibration of $\mathrm{Ni} \mathrm{SACs/N-C-based} \mathrm{sensor} \mathrm{for} \mathrm{NO} \mathrm{detection} \mathrm{was} \mathrm{per-}$ formed by adding a series of NO standard solution aliquots into the deaerated PBS. The electrode potential was held at $+0.80 \mathrm{~V}$ vs. $\mathrm{Ag} / \mathrm{AgCl}$ for amperometric detection.

The TOF $\left(\mathrm{h}^{-1}\right)$ for NO was calculated as using Eq. (4) as shown below:

$$
\mathrm{TOF}=\frac{I_{\text {product }} / N F}{m_{\text {cat }} \times \omega / M_{N i}} \times 3600
$$

$I_{\text {product: }}$ partial current for certain product, NO;

$N$ : the number of electron transferred for product formation, which is 1 for NO; F: Faradaic constant, $96485 \mathrm{C} \mathrm{mol}^{-1}$;

$m_{\text {cat }}:$ catalyst mass in the electrode, $\mathrm{g}$;

$\omega:$ Ni loading in the catalyst;

$M_{\mathrm{Ni}}$ : atomic mass of $\mathrm{Ni}, 58.69 \mathrm{~g} \mathrm{~mol}^{-1}$.

Human umbilical vein endothelial cell culture. Human umbilical vein endothelial cells (HUVECs) were purchased from ATCC (PCS-100-010) and were cultured using Endothelial medium (ScienCell,1001) at $37^{\circ} \mathrm{C}$ in a humidified incubator ( $95 \%$ air with $5 \% \mathrm{CO}_{2}$ ). Specially, the medium was made from $500 \mathrm{~mL}$ basal medium, $25 \mathrm{~mL}$ fetal bovine serum (FBS), $5 \mathrm{~mL}$ endothelial cell growth supplement (ECGS) and $5 \mathrm{~mL}$ penicillin/streptomycin solution. For cells experiment, HUVECs were cultivated on Ni SACs/N-C-based sensor which was kept in the incubator for $24 \mathrm{~h}$ to allow cells to adhesion. These loosely bounded HUVECs were washed with sterile PBS before electrochemically recording of NO release.

Real-time monitoring of NO release during cell mechano-transduction. In order to apply stretch strains to HUVECs, the Ni SACs/N-C-based sensor (active area, $0.5 \mathrm{~cm}^{2}$ ) cultured with HUVECs was carefully held with clamps on the transformable device and different stretching force was exerted on the sensor. The stretching and releasing time course of each strain was $5 \mathrm{~s}$ and $0.5 \mathrm{~s}$, respectively. The concentrations of both L-Arg and L-NAME (NOS inhibitor) added to HUVECs culture were $10.0 \mathrm{mM}$.

Computational details. In order to explore the geometrical and catalytic properties of the Ni SACs, several models with different curvatures were proposed for the Ni- $\mathrm{N}_{4}$ structure. These structures were geometrically optimized with the B3LYP hybrid density functional ${ }^{45,46}$ by using the Gaussian 03 program ${ }^{47}$. The Lanl2DZ pseudopotential for the Ni atoms and 6-31 G (d) basis sets for the other atoms were used in the calculations. All the structures were verified with vibrational analysis and no imaginary frequency was found. The zero-point energies and Gibbs free energies were also obtained in the vibrational analysis. After geometrical optimization, the XANES spectra of the models were calculated by using the ab initio multiple-scattering code JFEFF ${ }^{48}$. Full multiple scattering algorithm was used.

Reporting summary. Further information on research design is available in the Nature Research Reporting Summary linked to this article.

\section{Data availability}

The data that support the findings of this study are available from the authors upon reasonable request. The supplementary data are also available at public repositories (Identifier: DOI 10.17605/OSF.IO/7TZNY). The source data underlying Figs. 2a-d, 3a, b, d, and $4 \mathrm{~b}, \mathrm{c}$, e, $\mathrm{g}$ and Supplementary Figs. 2, 3a-c, 5a-c, 6, 7, 8, 10-12 are provided as a Source Data file.

Received: 5 January 2020; Accepted: 8 June 2020; Published online: 24 June 2020

\section{References}

1. Palmer, R. M. J. et al. Vascular endothelial cells synthesize nitric oxide from Largmine. Nature 333, 664-666 (1988).

2. Moncada, S. et al. Nitric oxide: physiology, pathophysiology, and pharmacology. Pharmacol. Rev. 43, 109-142 (1991). 
3. Ignarro, L. J. et al. Endothelium-derived relaxing factor produced and released from artery and vein is nitric oxide. Proc. Natl Acad. Sci. USA 84, 9265-9269 (1987).

4. Xiao, T. F. et al. In vivo analysis with electrochemical sensors and biosensors. Anal. Chem. 89, 300-313 (2017).

5. Jiang, S. et al. Real-time electrical detection of nitric oxide in biological systems with sub-nanomolar sensitivity. Nat. Commun. 4, 2225 (2013).

6. Hsieh, H. J. et al. Shear-induced endothelial mechanotransduction: the interplay between reactive oxygen species (ROS) and nitric oxide (NO) and the pathophysiological implications. J. Biomed. Sci. 21, 1-15 (2014).

7. Liu, Y. L. et al. Stretchable electrochemical sensor for real-time monitoring of cells and tissues. Angew. Chem. Int. Ed. 55, 4537-4541 (2016).

8. Zhao, X. et al. Fabrication of a flexible and stretchable nanostructured gold electrode using a facile ultraviolet-irradiation approach for the detection of nitric oxide released from cells. Anal. Chem. 90, 7158-7163 (2018).

9. Li, J. L. et al. Au nanoparticles-3D graphene hydrogel nanocomposite to boost synergistically in situ detection sensitivity toward cell-released nitric oxide. ACS Appl. Mater. Interfaces 7, 2726-2734 (2015).

10. Liu, L. Y. et al. A stretchable electrochemical sensor for inducing and monitoring cell mechanotransduction in real time. Angew. Chem. Int. Ed. 56, 9454-9458 (2017).

11. Wang, Y. W. et al. Stretchable and photocatalytically renewable electrochemical sensor based on sandwich nanonetworks for real-time monitoring of cells. Anal. Chem. 90, 5977-5981 (2018).

12. Qiao, B. T. et al. Single-atom catalysis of $\mathrm{CO}$ oxidation using $\mathrm{Pt}_{1} / \mathrm{FeO}_{\mathrm{x}}$. Nat. Chem. 3, 634-641 (2011).

13. Yang, X. F. et al. Single-atom catalysts: a new frontier in heterogeneous catalysis. Acc. Chem. Res. 46, 1740-1748 (2013).

14. Chen, Y. J. et al. Single-atom catalysts: synthetic strategies and electrochemical applications. Joule 2, 1242-1264 (2018).

15. Wang, A. et al. Heterogeneous single-atom catalysis. Nat. Rev. Chem. 2, 65 (2018).

16. Jiang, K. et al. Highly selective oxygen reduction to hydrogen peroxide on transition metal single atom coordination. Nat. Commun. 10, 3997 (2019).

17. Cheng, N. C. et al. Platinum single-atom and cluster catalysis of the hydrogen evolution reaction. Nat. Commun. 7, 13638 (2016).

18. Mao, J. J. et al. Accelerating water dissociation kinetics by isolating cobalt atoms into ruthenium lattice. Nat. Commun. 9, 4958 (2018).

19. Zhu, C. Z. et al. Single-atom electrocatalysts. Angew. Chem. Int. Ed. 56, 13944-13960 (2017).

20. Cheng, N. et al. Single-atom nanozyme based on nanoengineered Fe-N-C catalyst with superior peroxidase-like activity for ultrasensitive bioassays. Small 15, 1901485 (2019).

21. $\mathrm{Wu}, \mathrm{Y}$. et al. Oxidase-like Fe-N-C single-atom nanozymes for the detection of acetylcholinesterase activity. Small 15, 1903108 (2019).

22. $\mathrm{Ma}, \mathrm{W}$. J. et al. A single-atom $\mathrm{Fe}-\mathrm{N}_{4}$ catalytic site mimicking bifunctional antioxidative enzymes for oxidative stress cytoprotection. Chem. Commun. $\mathbf{5 5}$, 159-162 (2019).

23. Jiao, L. et al. When nanozymes meet single-atom catalysis. Angew. Chem. Int. Ed. 132, 2585-2596 (2020).

24. Yao, L. L. et al. Single-atom enzyme functionalized solution-gated graphene transistor for real-time detection of mercury ion. ACS Appl. Mater. Interfaces 56, 13944-13960 (2020).

25. Hou, H. F. et al. Single-atom electrocatalysis: a new approach to in vivo electrochemical biosensing. Sci. China Chem. 62, 1720-1724 (2019).

26. Gu, W. L. et al. Single-atom iron boosts electrochemiluminescence. Angew. Chem. Int. Ed. 59, 3534-3538 (2020).

27. Wang, J. et al. Highly active graphene oxide-supported cobalt single-ion catalyst for chemiluminescence reaction. Anal. Chem. 89, 13518-13523 (2017).

28. Huo, M. F. et al. Nanocatalytic tumor therapy by single-atom catalysts. ACS Nano 13, 2643-2653 (2019).

29. Liu, W. G. et al. A durable nickel single-atom catalyst for hydrogenation reactions and cellulose valorization under harsh conditions. Angew. Chem. Int. Ed. 57, 7071-7075 (2018).

30. Cao, L. L. et al. Dynamic oxygen adsorption on single-atomic ruthenium catalyst with high performance for acidic oxygen evolution reaction. Nat. Commun. 10, 4849 (2019).

31. Li, F. Y. et al. Styrene hydrogenation performance of Pt nanoparticles with controlled size prepared by atomic layer deposition. ACS Catal. 5, 544-552 (2015).

32. Zhang, M. N. et al. Rational design of surface/interface chemistry for quantitative in vivo monitoring of brain chemistry. Acc. Chem. Res. 45 533-543 (2012).

33. Zheng, D. Y. et al. Sensing of nitric oxide using a glassy carbon electrode modified with an electrocatalytic film composed of dihexadecyl hydrogen phosphate, platinum nanoparticles, and acetylene black. Microchim. Acta 176, 49-55 (2012)
34. Wang, Y. Z. et al. A multiwall carbon nanotubes film modified carbon fiber ultramicroelectrode for the determination of nitric oxide radical in liver mitochondria. Bioelectrochemistry 65, 135-142 (2005).

35. $\mathrm{Xu}, \mathrm{H}$. Y. et al. Iron phthalocyanine decorated nitrogen-doped graphene biosensing platform for real-time detection of nitric oxide released from living cells. Anal. Chem. 90, 4438-4444 (2018).

36. Bhat, S. A. et al. Self-assembled AuNPs on sulphur-doped graphene: A dual and highly efficient electrochemical sensor for nitrite $\left(\mathrm{NO}_{2}{ }^{-}\right)$and nitric oxide (NO). N. J. Chem. 41, 8347-8358 (2017).

37. Vinu Mohan, A. M. et al. Electrochemical codeposition of gold particle-poly (2-(2-pyridyl) benzimidazole) hybrid film on glassy carbon electrode for the electrocatalytic oxidation of nitric oxide. Sens. Actuators, B 196, 406-412 (2014).

38. Harrison, D. G. et al. Endothelial mechanotransduction, nitric oxide and vascular inflammation. J. Intern. Med. 259, 351-363 (2006).

39. Birukov, K. G. et al. Cyclic stretch, reactive oxygen species, and vascular remodeling. Antioxid. Redox, Sign, 11, 1651-1667 (2009).

40. Ali, M. H. et al. Mitochondrial requirement for endothelial responses to cyclic strain: Implications for mechanotransduction. Am. J. Physiol. Lung Cell. Mol. Physiol. 287, 486-496 (2004).

41. Cheng, J. J. et al. Cyclic strain-induced reactive oxygen species involved in ICAM-1 gene induction in endothelial cells. Hypertension 31, 125-130 (1998).

42. Matsushita, H. et al. Cyclic strain induces reactive oxygen species production via an endothelial NAD(P)H oxidase. J. Cell. Biochem. 81, 99-106 (2001).

43. Hahn, C. et al. Mechanotransduction in vascular physiology and atherogenesis. Nat. Rev. Mol. Cell Biol. 10, 53-62 (2009).

44. Liu, J. et al. Extension of the stöber method to the preparation of monodisperse resorcinol-formaldehyde resin polymer and carbon spheres. Angew. Chem. Int. Ed. 50, 5947-5951 (2011).

45. Becke,A. D. et al. Density-functional thermochemistry. III. The role of exact exchange. J. Chem. Phys. 98, 5648-5652 (1993).

46. Lee, C. et al. Development of the colle-salvetti correlation-energy formula into a functional of the electron density. Phys. Rev. B 37, 785-789 (1988).

47. Frisch, M. J. et al. Gaussian03; (Gaussian Inc: Wallingford, CT, USA, 2003).

48. Index of /jfeff-9.7.1. http://leonardo.phys.washington.edu/jfeff-9.7.1/ (2014).

\section{Acknowledgements}

This work was supported by the National Natural Science Foundation of China (No. 21575090 for Y.L.; Nos. 21790390 and 21790391 for L.M.; No. 21971002 for J.M.; Nos 21775151 and 21790053 for P.Y.), the National Key Research and Development Project (Grant No. 2018YFE0200800), the National Basic Research Program of China (Grant Nos. 2016YFA0200104), the Strategic Priority Research Program of Chinese Academy of Sciences (XDB30000000), the Beijing Nova Program of Science and Technology (Z191100001119108), Chinese Academy of Sciences (QYZDJ-SSW-SLH030), High-level Teachers in Beijing Municipal Universities in the Period of 13th Five year Plan (CIT\&TCD20190330), Scientific Research Project of Beijing Educational Committee (KM201810028008) and Youth Innovative Research Team of Capital Normal University.

\section{Author contributions}

L.M., Y.L., and J.M. conceived the idea for the project. J.M. and W.W. conducted material synthesis and structural characterizations. W.C. conducted XAFS measurements. M.Z. and Y.J. designed and performed catalytic performance testing, and cell studies. P.Y. and Y.J. analyzed the data; G.W. performed DFT calculations. M.Z., J.M., Y.L., and Y.J. drafted the manuscript, Y.J. and L.M. finalized the manuscript. M.Z. and Y.J. contributed equally to this work. All authors discussed and commented on the manuscript.

\section{Competing interests}

The authors declare no competing interests.

\section{Additional information}

Supplementary information is available for this paper at https://doi.org/10.1038/s41467 020-17018-6.

Correspondence and requests for materials should be addressed to Y.L., J.M. or L.M.

Peer review information Nature Communications thanks Wei-Hua Huang, Xijun Wang and the other anonymous reviewer(s) for their contribution to the peer review of this work. Peer reviewer reports are available.

Reprints and permission information is available at http://www.nature.com/reprints

Publisher's note Springer Nature remains neutral with regard to jurisdictional claims in published maps and institutional affiliations. 
(c) (i) Open Access This article is licensed under a Creative Commons Attribution 4.0 International License, which permits use, sharing, adaptation, distribution and reproduction in any medium or format, as long as you give appropriate credit to the original author(s) and the source, provide a link to the Creative Commons license, and indicate if changes were made. The images or other third party material in this article are included in the article's Creative Commons license, unless indicated otherwise in a credit line to the material. If material is not included in the article's Creative Commons license and your intended use is not permitted by statutory regulation or exceeds the permitted use, you will need to obtain permission directly from the copyright holder. To view a copy of this license, visit http://creativecommons.org/ licenses/by/4.0/.

(C) The Author(s) 2020 